\title{
Soliton Dynamics of Magnetization Driven by a Magnetic Field in Uniaxial Anisotropic Ferromagnet
}

\author{
Zhong-Xi Zheng ${ }^{1}$, Qiu-Yan $\mathrm{Li}^{2}$, Zai-Dong $\mathrm{Li}^{2}$, and Ting-Dun Wen ${ }^{1}$ \\ ${ }^{1}$ Department of Physics, North University of China, Taiyuan 030051, China \\ ${ }^{2}$ Department of Applied Physics, Hebei University of Technology, Tianjin 300130, China
}

Received on 9 October, 2006

\begin{abstract}
We study the nonlinear magnetic excitation in an anisotropic ferromagnet with a magnetic field. In the long wave approximation, the Landau-Lifschitz equation with easy axis anisotropy is transformed into the nonlinear Schrödinger type. By means of a straightforward Darboux transformation we obtain the one- and two-soliton solutions of uniaxial anisotropic ferromagnet. From a careful analysis for the asymptotic behavior of twosoliton solution we find that the collision between two magnetic solitons is elastic. This will be very helpful to understand the significant nature of the interactions between solitons in the future.
\end{abstract}

Keywords: Soliton; Darboux transformation; Soliton interactions

The Heisenberg model of spin-spin interactions can be considered as the starting point for understanding the complex magnetic structures in solid physics. In particular, it explains the existence of ferromagnetism and antiferromagnetism at temperature below the Curie temperature. This model has attracted considerable attentions in nonlinear science and condensed-matter physics [1]. The concept of soliton in spin chain which exhibits both coherent and chaotic structures depending on the nature of the magnetic interactions [1-3] has been studied for decades. In the present time soliton in quasi one-dimensional magnetic systems is no longer a theoretical concept but can be probed by neutron inelastic scattering [4], nuclear magnetic resonance [5], and electron spin resonance [6]. The magnetic soliton [7], which describes localized magnetization, is an important excitation in the classical Heisenberg spin chain. In particular, the continuum limit for the nonlinear dynamics of magnetization in the classical ferromagnet is governed by the Landau-Lifschitz (L-L) equation [8]. This equation governs a classical nonlinear dynamically system with novel properties. In a onedimensional case, some types of L-L equation is complete integrable. The isotropic case has been studied in various aspects $[9,10]$, and the construction of soliton solutions of L-L equation with an easy axis is also discussed [11]. It is worth to noted that the inverse scattering transformation $[10,12]$ is a useful method to solve the L-L equation. On the other hand great efforts [13] are also devoted to construct the soliton solution by means of the Darboux transformation [14-17].

In the recent years, considerable attentions have been devoted to the study of soliton interactions in nonlinear science. However, the soliton collisions in spin chain is not fully explored. In this paper, we investigate soliton interactions of uniaxial anisotropic ferromagnet with an external magnetic field. By transforming the L-L equation with an easy-axis into an equation of the nonlinear Schrôdinger (NLS) type we obtain the one- and two-soliton solutions by using the Darboux transformation.

In the classical limit, the dynamics of spin chain is governed by the magnetization vector $\boldsymbol{M}=\left(M_{x}, M_{y}, M_{z}\right)$. The energy function including the exchange energy, anisotropic energy and the Zeeman energy can be written as [7]

$$
E=\frac{1}{2} \int\left[\alpha\left(\frac{\partial M}{\partial x}\right)^{2}-\beta M_{z}^{2}-M \cdot B\right] d^{3} x
$$

where $\alpha$ is the exchange constant and $\beta$ is the uniaxial anisotropic constant, $\beta>0$ corresponds to easy-axis anisotropy while $\beta<0$ corresponds to easy-plane type. The dynamics of the magnetization vector $M(x, t)$ is determined by the following equation

$$
\frac{\partial M}{\partial t}=\frac{2 \mu_{0}}{\hbar} M \times \frac{\delta E}{\delta M},
$$

where $\mu_{0}$ is Bohr magneton. Substituting Eq. (1) into Eq. (2), we can get the $\mathrm{L}-\mathrm{L}$ equation

$$
\frac{\hbar}{2 \mu_{0}} \frac{\partial M}{\partial t}=-\alpha M \times \frac{\partial^{2} M}{\partial x^{2}}-\beta M \times e_{3}\left(M \cdot e_{3}\right)-\mu_{0} M \times B,
$$

where $e_{3}$ is the unit vector along the $z$-axis, and $\boldsymbol{B}=$ $(0,0, B(t))$. Taking into account the integral of motion $\boldsymbol{M}^{2} \equiv$ $M_{0}^{2}=$ constant, and introducing a single function $\Psi$, instead of two independent components of $M$,

$$
\Psi=m_{x}+i m_{y}, m_{z}=\sqrt{1-|\Psi|^{2}},
$$

where $\mathbf{m} \equiv\left(m_{x}, m_{y}, m_{z}\right)=M / M_{0}, M_{0}$ is the equilibrium magnetization. Thus Eq. (3) becomes

$$
\begin{aligned}
& \frac{i \hbar}{2 \mu_{0}} \frac{\partial \Psi}{\partial t}-\alpha M_{0}\left(m_{z} \frac{\partial^{2}}{\partial x^{2}} \Psi-\Psi \frac{\partial^{2}}{\partial x^{2}} m_{z}\right) \\
= & -\left[\beta M_{0} m_{z}+B(t)\right] \Psi .
\end{aligned}
$$

In ground state, vector $M$ directs along the anisotropy axis $e_{3}$. Now we consider the low excitation, namely, the small deviations of $M$ from $e_{3}$ which correspond to the values $|\Psi| \ll 1$, then $m_{z} \approx 1-1 / 2|\Psi|^{2}$. In the long-wavelength approximation and the case $\beta>0$, Eq. (5) may be simplified by keeping only the nonlinear terms of the order of the magnitude of $|\psi|^{2} \psi$. As a result, we have the following dimensionless Schrödinger equation: 


$$
i \frac{\partial \Psi}{\partial t}-\frac{1}{2} \frac{\partial^{2} \Psi}{\partial x^{2}}-|\Psi|^{2} \Psi+2\left[1+\frac{B(t)}{\beta M_{0}}\right] \Psi=0 .
$$

For the convenience we have rescaled the space $x$ and the time $t$ by the $2 l_{0}$ and $1 / \omega_{0}$, where $l_{0}=\sqrt{\alpha / \beta}$ is the characteristic magnetic length and $\omega_{0}=\beta \mu_{0} M_{0} / \hbar$ is the homogeneous ferromagnetic resonance frequency.

In the following, we use the Darboux transformation to get exact soliton solutions of Eq. (6). By employing AblowitzKaup-Newell-Segur technique one can construct the lax representation for Eq. (6) as follows

$$
\frac{\partial}{\partial x} \psi=L \psi, \quad \frac{\partial}{\partial t} \psi=M \psi
$$

where $\psi=\left(\begin{array}{ll}\psi_{1} & \psi_{2}\end{array}\right)^{T}$, the superscript " $T$ " denotes the matrix transpose. The lax pairs $L$ and $M$ are given in the forms

$$
\begin{aligned}
L & =\lambda J+P \\
M & =\left[-i \lambda^{2}+\alpha_{2}(t)\right] J-i \lambda P+\frac{1}{2} i\left(P^{2}+\frac{\partial}{\partial x} P\right) J,
\end{aligned}
$$

with

$$
J=\left(\begin{array}{ll}
1 & 0 \\
0 & -1
\end{array}\right), P=\left(\begin{array}{ll}
0 & \Psi \\
-\Psi & 0
\end{array}\right), \alpha_{2}(t)=i\left(1+\frac{B}{\beta M_{0}}\right),
$$

where the overbar denotes the complex conjugate. Thus the Eq. (6) can be recovered from the compatibility condition $\frac{\partial}{\partial t} L-\frac{\partial}{\partial x} M+[L, M]=0$. Based on the Lax pair (7), we can obtain the general one- and two-soliton solution by using a straightforward Darboux transformation [18, 19].

Consider the following transformation

$$
\psi[1]=(\lambda I-S) \psi, S=K \Lambda K^{-1}, \Lambda=\operatorname{diag}\left(\lambda_{1}, \lambda_{2}\right),
$$

where $K$ satisfies

$$
\frac{\partial}{\partial x} K=J K \Lambda+P K
$$

Letting

$$
\frac{\partial}{\partial x} \psi[1]=L_{1} \psi[1]
$$

where $L_{1}=\lambda J+P_{1}, P_{1}=\left(\begin{array}{lr}0 & \Psi_{1} \\ -\bar{\Psi}_{1} & 0\end{array}\right)$, and with the help of Eqs. (7), (8) and (9), we obtain the Darboux transformation for Eq. (6) from Eq. (10) in the form

$$
\Psi_{1}=\Psi+[J, S]
$$

It is easy to verify that, if $\psi=\left(\begin{array}{ll}\psi_{1} & \psi_{2}\end{array}\right)^{T}$ is a eigenfunction of Eq. (7) corresponding to the eigenvalue $\lambda=\lambda_{1}$, then $\left(-\bar{\psi}_{2} \bar{\psi}_{1}\right)^{T}$ is also the eigenfunction, while with the eigenvalue $-\bar{\lambda}_{1}$. Hence if taking the following notations

$$
K=\left(\begin{array}{ll}
\psi_{1} & -\bar{\psi}_{2} \\
\psi_{2} & \bar{\psi}_{1}
\end{array}\right), \Lambda=\left(\begin{array}{cc}
\lambda_{1} & 0 \\
0 & -\bar{\lambda}_{1}
\end{array}\right)
$$

which ensures that Eq. (9) is held, we can obtain

$$
S_{s l}=-\bar{\lambda}_{1} \delta_{s l}+\left(\lambda_{1}+\bar{\lambda}_{1}\right) \frac{\psi_{s} \bar{\psi}_{l}}{\psi^{T} \bar{\psi}}, s, l=1,2
$$

where $\psi^{T} \bar{\psi}=\left|\psi_{1}\right|^{2}+\left|\psi_{2}\right|^{2}$, and Eq. (11) becomes

$$
\Psi_{1}=\Psi+2\left(\lambda_{1}+\bar{\lambda}_{1}\right) \frac{\psi_{1} \bar{\psi}_{2}}{\psi^{T} \bar{\psi}}
$$

where $\psi$ is the eigenfunction of Eq. (7) corresponding to the eigenvalue $\lambda_{1}$ for the solution $\Psi$. Thus by solving the Eq. (7) which is a first-order linear differential equation, we can generate a new solution $\Psi_{1}$ of the Eq. (6) from a known solution $\Psi$ which is usually called "seed" solution.

To obtain exact $N$-order solution of Eq. (6), we firstly rewrite the Darboux transformation in Eq. (14) as in the form

$$
\Psi_{1}=\Psi+2\left(\lambda_{1}+\bar{\lambda}_{1}\right) \frac{\Psi_{1}\left[1, \lambda_{1}\right] \bar{\Psi}_{2}\left[1, \lambda_{1}\right]}{\psi\left[1, \lambda_{1}\right]^{T} \bar{\psi}\left[1, \lambda_{1}\right]},
$$

where $\psi[1, \lambda]=\left(\psi_{1}[1, \lambda], \psi_{2}[1, \lambda]\right)^{T}$ denotes the eigenfunction of Eq. (7) corresponding to eigenvalue $\lambda$. Then repeating above the procedure for $N$ times, we can obtain the exact $N$ order solution

$$
\Psi_{N}=\Psi+2 \sum_{n=1}^{N}\left(\lambda_{n}+\bar{\lambda}_{n}\right) \frac{\Psi_{1}\left[n, \lambda_{n}\right] \bar{\Psi}_{2}\left[n, \lambda_{n}\right]}{\psi\left[n, \lambda_{n}\right]^{T} \bar{\Psi}\left[n, \lambda_{n}\right]},
$$

where

$$
\begin{aligned}
& \psi[n, \lambda]=(\lambda-S[n-1]) \cdots(\lambda-S[1]) \psi[1, \lambda], \\
& S_{s l}\left[j^{\prime}\right]=-\bar{\lambda}_{j^{\prime}} \delta_{s l}+\left(\lambda_{j^{\prime}}+\bar{\lambda}_{j^{\prime}}\right) \frac{\psi_{s}\left[j^{\prime}, \lambda_{j^{\prime}}\right] \bar{\psi}_{l}\left[j^{\prime}, \lambda_{j^{\prime}}\right]}{\psi\left[j^{\prime}, \lambda_{j^{\prime}}\right]^{T} \bar{\psi}\left[j^{\prime}, \lambda_{j^{\prime}}\right]},
\end{aligned}
$$

here $\psi\left[j^{\prime}, \lambda\right]$ is the eigenfunction corresponding to $\lambda_{j^{\prime}}$ for $\Psi_{j^{\prime}-1}$ with $\Psi_{0} \equiv \Psi$ and $s, l=1,2, j^{\prime}=1,2, \cdots, n-1, n=$ $2,3, \cdots, N$. Thus if choosing a "seed" as the basic initial solution, by solving linear characteristic equation system (7), one can construct a set of new solutions for Eq. (6) by employing the formula (16).

Taking the spectral parameter $\lambda_{1}=\mu_{1}+i v_{1}$ we get the onesoliton solution from Eqs. (16) and (4)

$$
m_{x}=\frac{2 \mu_{1} \cos \Phi_{1}}{\cosh \Theta_{1}}, m_{y}=\frac{2 \mu_{1} \sin \Phi_{1}}{\cosh \Theta_{1}}, m_{z}=\sqrt{1-\frac{4 \mu_{1}^{2}}{\cosh ^{2} \Theta_{1}}},
$$

where

$$
\begin{aligned}
\Theta_{1} & =-2 \mu_{1}\left(x+2 v_{1} t-\frac{x_{0}^{\prime}}{\mu_{1}}\right) \\
\Phi_{1} & =2 v_{1}\left[x-\frac{1}{v_{1}}\left(\mu_{1}^{2}-v_{1}^{2}\right) t\right. \\
& \left.+\frac{1}{v_{1}} \int\left(1+\frac{B(t)}{\beta M_{0}}\right) d t-x_{0}^{\prime \prime} / v_{1}\right]
\end{aligned}
$$

The parameters $x_{0}^{\prime} / \mu_{1}$ and $x_{0}^{\prime \prime} / v_{1}$ represent the initial center position and initial phase. To show the physical significance 
of our solutions, it is useful to give the parameter dependence of polar angle of the magnetization vector such that $\mathbf{m}=(\sin \theta \cos \varphi, \sin \theta \sin \varphi, \cos \theta)$, namely the $z$-axis is the polar axis in a spherical coordinate. From Eq. (17) we have that

$$
\begin{aligned}
\theta= & \arccos \left(\sqrt{1-\frac{4 \mu_{1}^{2}}{\cosh ^{2} \Theta_{1}}}\right) \\
\varphi= & 2 v_{1}\left[x-\frac{1}{v_{1}}\left(\mu_{1}^{2}-v_{1}^{2}\right) t\right. \\
& \left.+\frac{1}{v_{1}} \int\left(1+\frac{B(t)}{\beta M_{0}}\right) d t-x_{0}^{\prime \prime} / v_{1}\right]
\end{aligned}
$$

The expression (18) describes a magnetization precession characterized by four real parameters: velocity $2 v_{1}$, frequency $\frac{1}{v_{1}}\left[\left(\mu_{1}^{2}-v_{1}^{2}\right)-1-B(t) /\left(\beta M_{0}\right)\right]$, initial center position $x_{0}^{\prime} / \mu_{1}$ and initial phase $x_{0}^{\prime \prime} / \nu_{1}$. Therefore, we can see that the external magnetic field contribute to precession frequency only.

The magnetic soliton collision is an interesting phenomenon in spin dynamics. To this purpose we should obtain the two-soliton solution of Eq. (6). Taking the spectral parameter $\lambda_{j}=\mu_{j}+i v_{j}, j=1,2$, from the expressions (16) and (4) we obtain the two-soliton solution of magnet as

$$
\begin{aligned}
m_{x}= & 2 \gamma \frac{1}{\Delta}\left[\mu_{2}\left(f_{1} \cos \Phi_{2}+g_{1} \sin \Phi_{2}\right)\right. \\
& \left.+\mu_{1}\left(f_{2} \cos \Phi_{1}-g_{2} \sin \Phi_{1}\right)\right], \\
m_{y}= & 2 \gamma \frac{1}{\Delta}\left[\mu_{2}\left(f_{1} \sin \Phi_{2}-g_{1} \cos \Phi_{2}\right)\right. \\
& \left.+\mu_{1}\left(f_{2} \sin \Phi_{1}+g_{2} \cos \Phi_{1}\right)\right], \\
m_{z}= & \sqrt{1-\left(2 \gamma \frac{1}{\Delta}\right)^{2} \Gamma,}
\end{aligned}
$$

where

$$
\begin{aligned}
& f_{1}=\left(\gamma-2 \mu_{1} \cos \phi_{0}\right) \cosh \Theta_{1}, g_{1}=2 \mu_{1} \sin \phi_{0} \sinh \Theta_{1}, \\
& f_{2}=\left(\gamma-2 \mu_{2} \cos \phi_{0}\right) \cosh \Theta_{2}, g_{2}=2 \mu_{2} \sin \phi_{0} \sinh \Theta_{2},
\end{aligned}
$$

$$
\begin{aligned}
\Delta= & \gamma^{2} \cosh \Theta_{1} \cosh \Theta_{2} \\
& -2 \mu_{1} \mu_{2}\left[\cosh \left(\Theta_{1}+\Theta_{2}\right)+\cos \left(\Phi_{1}-\Phi_{2}\right)\right] \\
\Gamma= & \mu_{2}^{2}\left(f_{1}^{2}+g_{1}^{2}\right)+\mu_{1}^{2}\left(f_{2}^{2}+g_{2}^{2}\right) \\
& +2 \mu_{1} \mu_{2}\left[\left(f_{1} f_{2}-g_{1} g_{2}\right) \cos \left(\Phi_{1}-\Phi_{2}\right)\right. \\
& \left.-\left(f_{1} g_{2}+f_{2} g_{1}\right) \sin \left(\Phi_{1}-\Phi_{2}\right)\right] .
\end{aligned}
$$

where $\gamma=\left|\lambda_{2}+\bar{\lambda}_{1}\right|, \phi_{0}=\arg \left(\lambda_{2}+\bar{\lambda}_{1}\right)$, and the parameters $\Theta_{j}$ and $\Phi_{j}, j=1,2$, are defined as

$$
\begin{aligned}
\Theta_{j} & =-2 \mu_{j}\left(x+2 v_{j} t-x_{0, j}^{\prime} / \mu_{j}\right) \\
\Phi_{j} & =2 v_{j}\left[x-\frac{1}{v_{j}}\left(\mu_{j}^{2}-v_{j}^{2}\right) t\right. \\
& \left.+\frac{1}{v_{j}} \int\left(1+B(t) /\left(\beta M_{0}\right)\right) d t-x_{0, j}^{\prime \prime} / v_{j}\right] .
\end{aligned}
$$

The solution (19) describes a general scattering process of two solitary waves with different center velocities $2 v_{1}$ and $2 v_{2}$, different phases $\Phi_{1}$ and $\Phi_{2}$. Before collision, they move towards each other, one with velocity $2 v_{1}$ and shape variation frequency $\Omega_{1}=\frac{1}{v_{1}}\left[\left(\mu_{1}^{2}-v_{1}^{2}\right)-1-B(t) /\left(\beta M_{0}\right)\right]$ and the other with $2 v_{2}$ and $\Omega_{2}=\frac{1}{v 2}\left[\left(\mu_{2}^{2}-v_{2}^{2}\right)-1-B(t) /\left(\beta M_{0}\right)\right]$. In order to understand the nature of two-soliton interaction, we analyze the asymptotic behavior of two-soliton solution (19). Asymptotically, the two-soliton waves (19) can be written as a combination of two one-soliton waves (17) with different amplitudes and phases. The asymptotic form of twosoliton solution in limits $t \rightarrow-\infty$ and $t \rightarrow \infty$ is similar to that of the one-soliton solution (17). In order to analyze the asymptotic behavior of two-soliton solutions (19) we consider the following asymptotic discussion for $\Theta_{1} \sim 0, \Theta_{2} \sim \pm \infty$, as $t \rightarrow \pm \infty$; and (ii) $\Theta_{2} \sim 0, \Theta_{1} \sim \mp \infty$, as $t \rightarrow \pm \infty$. This leads to the following asymptotic forms for the two-soliton solution.

(i) Before collision-namely, the case of limit $t \rightarrow-\infty$.

(a) Soliton $1\left(\Theta_{1} \sim 0, \Theta_{2} \rightarrow-\infty\right)$.

$$
\begin{aligned}
& m_{x} \rightarrow 2 \mu_{1} \frac{\cos \left(\Phi_{1}-\phi_{2}\right)}{\cosh \left(\Theta_{1}+\chi_{0}\right)}, m_{y} \rightarrow 2 \mu_{1} \frac{\sin \left(\Phi_{1}-\phi_{2}\right)}{\cosh \left(\Theta_{1}+\chi_{0}\right)}, \\
& m_{z} \rightarrow \sqrt{1-\frac{4 \mu_{1}^{2}}{\cosh ^{2}\left(\Theta_{1}+\chi_{0}\right)}} .
\end{aligned}
$$

(b) Soliton $2\left(\Theta_{2} \sim 0, \Theta_{1} \rightarrow+\infty\right)$.

$$
\begin{aligned}
& m_{x} \rightarrow 2 \mu_{2} \frac{\cos \left(\Phi_{1}-\phi_{1}\right)}{\cosh \left(\Theta_{2}-\chi_{0}\right)}, m_{y} \rightarrow 2 \mu_{2} \frac{\sin \left(\Phi_{1}-\phi_{1}\right)}{\cosh \left(\Theta_{2}-\chi_{0}\right)}, \\
& m_{z} \rightarrow \sqrt{1-\frac{4 \mu_{2}^{2}}{\cosh ^{2}\left(\Theta_{2}-\chi_{0}\right)}} .
\end{aligned}
$$

(ii)After collision-namely, the case of limit $t \rightarrow+\infty$.

(a) Soliton $1\left(\Theta_{1} \sim 0, \Theta_{2} \sim+\infty\right)$

$$
\begin{aligned}
& m_{x} \rightarrow 2 \mu_{1} \frac{\cos \left(\Phi_{1}+\phi_{2}\right)}{\cosh \left(\Theta_{1}-\chi_{0}\right)}, m_{y} \rightarrow 2 \mu_{1} \frac{\sin \left(\Phi_{1}+\phi_{2}\right)}{\cosh \left(\Theta_{1}-\chi_{0}\right)}, \\
& m_{z} \rightarrow \sqrt{1-\frac{4 \mu_{1}^{2}}{\cosh ^{2}\left(\Theta_{1}-\chi_{0}\right)}} .
\end{aligned}
$$

(b) Soliton $2\left(\Theta_{2} \sim 0, \Theta_{1} \sim-\infty\right)$

$$
\begin{aligned}
& m_{x} \rightarrow 2 \mu_{2} \frac{\cos \left(\Phi_{1}+\phi_{1}\right)}{\cosh \left(\Theta_{2}+\chi_{0}\right)}, m_{y} \rightarrow 2 \mu_{2} \frac{\sin \left(\Phi_{1}+\phi_{1}\right)}{\cosh \left(\Theta_{2}+\chi_{0}\right)}, \\
& m_{z} \rightarrow \sqrt{1-\frac{4 \mu_{2}^{2}}{\cosh ^{2}\left(\Theta_{2}+\chi_{0}\right)}},
\end{aligned}
$$

where the parameters $\chi_{0}$ and $\phi_{j}, j=1,2$ in equations (20) to (23) are defined by

$$
\begin{aligned}
& \phi_{1}=\arctan \frac{-2 \mu_{1}\left(v_{1}-v_{2}\right)}{\left(\mu_{2}^{2}-\mu_{1}^{2}\right)+\left(v_{1}-v_{2}\right)^{2}} \\
& \phi_{2}=\arctan \frac{-2 \mu_{2}\left(v_{1}-v_{2}\right)}{\mu_{1}^{2}-\mu_{2}^{2}+\left(v_{1}-v_{2}\right)^{2}} \\
& \chi_{0}=\frac{1}{2} \ln \left[\frac{\left(\mu_{1}+\mu_{2}\right)^{2}+\left(v_{1}-v_{2}\right)^{2}}{\left(\mu_{1}-\mu_{2}\right)^{2}+\left(v_{1}-v_{2}\right)^{2}}\right] .
\end{aligned}
$$


From equations (20), (21), (22), and (23) we can see that there is no amplitude exchange among three components $m_{x}$, $m_{y}$ and $m_{z}$ for magnetization vector soliton 1 and soliton 2 during collision. However, from Eq. (20) to (23) one can see that there is a phase change $2 \phi_{2}\left(2 \phi_{1}\right)$ for the components $m_{x}$ and $m_{y}$ of the soliton 1(2), and the center position change $-2 \chi_{0}\left(+2 \chi_{0}\right)$ for the components $m_{z}$ for soliton 1(2) during collision. This interaction between two magnetic solitons is called elastic collision. The magnetic soliton solutions in Eqs. (17) and (19), which describes localized magnetization, is an important excitation in the Heisenberg spin chain. The significant importance of the study for the soliton is that it can travel over long distances with neither attenuation nor change of shape, since the dispersion is compensated by nonlinear effects. This type of the elastic soliton collision shows that the information held in each soliton will almost not be disturbed by each other in soliton propagation. These properties may have potential application in future quantum communication.
In conclusion, by transforming the L-L equation with an easy-axis into an equation of the nonlinear Schrődinger type we investigate the nonlinear magnetic excitations in an anisotropic ferromagnet with a magnetic field. From a straightforward Darboux transformation the one- and twosoliton solutions of uniaxial anisotropic ferromagnet with an external magnetic field are reported. Moreover, by analyzing carefully the asymptotic behavior of two-soliton solution we find that the collision between two magnetic solitons is elastic. This is very helpful to understand significant nature of the interactions between solitons in the future.

This work was supported the Natural Science Foundation of China No. 10647122 and 60476063, the Doctoral Foundation of Education Bureau of Hebei Province No. 2006110, the key subject construction project of Heibei Provincical University, and the Natural Science Foundation of Shanxi Province No. 20041011
[1] Y. S. Kivshar and B. A. Malomed, Rev. Mod. Phys. 61763 , (1989).

[2] S. E. Trullinger, V. E. Zakharov and V. L. Pokrovsky, Solitons (Elsevier, New York), (1986).

[3] M. J. Ablowitz and P. A. Clarkson Solitons, Nonliear Evoluation Equations and Inverse Scattering (Cambrige University Press, Cambrige), (1991).

[4] J. P. Boucher, R. Pynn, M. Remoissenet, L. P. Regnault, Y. Endoh, and J. P. Renard, Phys. Rev. Lett. 64 1557, (1990).

[5] J. P. Boucher and J. P. Renard, Phys. Rev. Lett. 45 486, (1980).

[6] T. Asano, H. Nojiri, Y. Inagaki, J. P. Boucher, T. Sakon, Y. Ajiro, and M. Motokawa, Phys. Rev. Lett. 84 5880, (2000).

[7] A. M. Kosevich, B. A. Ivanoy and A. S. Kovalev, Phys. Rep. 194 117, (1990).

[8] L. D. Landau and E. M. Lifschitz, Phys. Z. Sowjetunion 8 153, (1935).

[9] M. Laksmanan, Phys. Lett. A 61 53, (1977); J. Tjon and J. Wright, Phys. Rev. B 15 3470, (1977); T, Shimizu, J. Phys. Society of Japan $\mathbf{5 3}$ 507, (1984).

[10] L. A. Takhtajan, Phys. Lett. A 64 235, (1977).

[11] A. E. Bolovik, Sov. Phys.-JETP Lett. 28 629, (1978); G. R. W. Quispel and H. W. Capel, Physica A 117 76, (1983).

[12] H. C. Fogedby, J. Phys. A 13 1467, (1980); Z. Y. Chen, N. N. Huang and Z. Z. Liu, J. Phys. Condens. Matter 7 4533, (1995); Z. D. Li, Lu Li, W. M. Liu, J. Q. Liang and T. Ziman Phys. Rev.
E 68 036102, (2003); Z. D. Li, J. Q. Liang, Lu Li, and W. M. Liu, Phys. Rev. E 69 066611, (2004).

[13] H. J. Mikeska, Physica C 11 L29, (1978); K. A. Long and A. R. Bishop, J. Phys. A 12 1325, (1979).

[14] N. N. Huang, Z. Y. Chen, and Z. Z. Liu, Phys. Rev. Lett. 75 1395, (1995)

[15] W. M. Liu, Shu-Li Yang, Fu-Cho Pu, and Nian-Ning Huang, Z. Phys. B 103 105, (1997); W. M. Liu, Wu-Shou Zhang and Fu-Cho Pu, Phys. Rev. B 60 12893, (1999); W. M. Liu, B. Wu, X. Zhou, D. K. Campbell, S. T. Chui and Q. Niu, Phys. Rev. B 65 172416, (2002).

[16] Q. Y. Li, Z. W. Xie, L. Li, Z. D. Li, and J. Q. Liang, Annals of Physics 312, 128, (2004); Z. D. Li, Lu Li and J. Q. Liang, Chin. Phys. Lett. 20 39, (2003); Z. D. Li, Lu Li and J. Q. Liang, Chin. Phys. Lett. 21 443, (2004).

[17] X. J. Chen, L. J. Hou, and Wa Kun Lam, Chin. Phys. Lett. 22 $830,(2005)$

[18] V. B. Matveev and M. A. Salli, Darboux Transformations and Solitons, (Springer Series in Nonlinear Dynamics, Springer, Berlin), (1991).

[19] C. H. Gu, H. S. Hu, Z. X. Zhou, Darboux Transformation in Soliton Theory and its Geometric Applications (Shanghai Scientific and Technical Publishers, in Chinese), (1999). 